\title{
Tratamiento y reutilización del agua residual de lavadora
}

\section{Treatment and reuse of washer wastewater}

\author{
Efraín Sianca ${ }^{1}$, Grisneyly Castro ${ }^{1}$, Liz Him ${ }^{1}$, Marlenys Gómez ${ }^{1}$, Fabio Velarde ${ }^{2 *}$ \\ ${ }^{1}$ Licenciatura en Ingeniería Industrial - Centro Regional de Veraguas - Universidad Tecnológica de Panamá \\ ${ }^{2}$ Facultad de Ingeniería Mecánica - Centro Regional de Veraguas - Universidad Tecnológica de Panamá
}

Resumen En este artículo se presenta el diseño y funcionamiento de un sistema de tratamiento de agua residual de lavadora; el cual consiste en la recolección del agua que será tratada mediante el proceso de floculación y luego pasará a ser filtrada obteniendo así el agua limpia que será reutilizada para el siguiente proceso de lavado y otros usos en el hogar.

Palabras clave Agua residual, coagulación, filtración, floculante, reutilización.

\begin{abstract}
This article presents the design and operation of a wastewater treatment system for washing machines; which consists in the collection of the waste water which is then treated through the flocculation process and finally filtered obtaining clean water that could be reused for the next washing process or other uses in the home.
\end{abstract}

Keywords Waste water, coagulation, filtration, flocculant, reuse.

* Corresponding Author: fabiovelarde@hotmail.com

\section{Introducción}

Este proyecto se basa en el diseño de un sistema de tratamiento de aguas para los hogares. En los hogares uno de los principales procesos internos es el lavado de ropa, por esta razón el consumo de agua es bastante elevado. Debido a esto se puede decir que el diseño de este proyecto se enfoca en aprovechar y reutilizar el agua de la lavadora después de cada proceso de lavado, evitando así el desperdicio del recurso y a la vez generando una disminución en el consumo del agua.

\section{Marco teórico}

\subsection{Tratamiento de aguas residuales}

El tratamiento de aguas residuales consiste en una serie de procesos físicos, químicos y biológicos que tienen como fin eliminar los contaminantes presentes en el agua efluente del uso humano. En el mundo existen diferentes métodos y formas de realizar tratamientos para el agua, los cuales son implementados según el grado de contaminación que se presente [1].

El tratamiento conlleva los siguientes pasos:

$>$ Tratamiento primario: En esta etapa se busca eliminar los sólidos suspendidos por medio de métodos como la sedimentación, usando tratamientos físicos y fisicoquímicos. Normalmente se busca dejar el agua en reposo en tanques para realizar el proceso de sedimentación natural o por gravedad, también se pueden agregar productos químicos que ayudan a sedimentar los sólidos de manera más eficiente como los floculantes.

- Sedimentación: consiste en la separación, por la acción de la gravedad de las partículas suspendidas cuyo peso específico es mayor que el del agua y no pueden retenerse en las unidades de pretratamiento, por su finura o densidad, ni pueden separarse por flotación [2].

Tratamientos Químicos: Son procesos que permiten realizar tratamientos a las aguas de manera más eficiente y rápida. Consisten usualmente en adicionar reactivos químicos que logran realizar ciertas actividades de separación de sólidos suspendidos en el agua para facilitar la sedimentación de los mismos.

- Coagulación: Es un proceso de desestabilización química de las partículas coloidales que se producen al neutralizar las fuerzas que los mantienen separados, por medio de la adición de los coagulantes químicos y la aplicación de la energía de mezclado [3].

- Floculación: La floculación es el proceso que sigue a la coagulación, que consiste en la agitación de la masa coagulada que sirve para permitir el crecimiento y aglomeración de los flóculos recién formados con la finalidad de aumentar el tamaño y peso necesarios para sedimentar con facilidad [4]. 


\subsection{Sistema de reutilización}

La reutilización de las aguas es una forma de volver a utilizar el recurso hídrico varias veces en procesos cotidianos que no requieran de aguas muy potabilizadas.

De acuerdo a lo anterior, podemos entender que la reutilización del agua en un proceso cíclico en el cual se vuelva a dar uso al recurso hídrico mediante algún tipo de sistema cerrado.

\section{Materiales}

Tabla 1. Materiales utilizados en la construcción del sistema.

\begin{tabular}{|c|c|}
\hline Material & Cantidad \\
\hline Tanque de 20 litros & 1 \\
\hline Tanque chico & 1 \\
\hline Flanyer de $1 / 2 "$ & 1 \\
\hline Válvula de 1/2" de PVC & 2 \\
\hline Conector macho de $1 / 2 "$ de PVC & 4 \\
\hline Conector hembra de $1 / 2 "$ de PVC & 4 \\
\hline Angulo de $90^{\circ}$ de $1 / 2 "$ de PVC & 2 \\
\hline Tubo PVC de $1 / 2 "$ & $60 \mathrm{~cm}$ \\
\hline Tubo PVC de 4" & $50 \mathrm{~cm}$ \\
\hline Tapa PVC para tubo de 4" & 2 \\
\hline Cinta de teflón & Un rollo \\
\hline Tapa de plástico grande & 1 \\
\hline Llave de jardín PVC & 1 \\
\hline Carbón vegetal & 1 bolsa \\
\hline \multicolumn{2}{|l|}{ Algodón } \\
\hline Colador & 1 \\
\hline Segueta & 1 \\
\hline Tijeras & 1 \\
\hline Lija & 1 \\
\hline Angulo de aluminio & 1 \\
\hline Alumbre & 20 a $50 \mathrm{~g}$ \\
\hline
\end{tabular}

\section{Descripción del sistema}

\subsection{Descripción física}

El sistema consta de dos tanques; uno de almacenamiento de agua (tanque 1), del cual se conecta una tubería que lleva a la válvula conectada con el filtro y del filtro a la llave; y otro de almacenamiento de residuos (tanque 2), que se encuentra conectado debajo del tanque de almacenamiento de agua.

\subsection{Descripción del proceso}

El agua desechada de la lavadora es acumulada en el tanque 1 donde se le adiciona el coagulante, se mezcla de tres a cinco minutos y deja reposar durante 30 a 60 minutos para que los sólidos suspendidos se sedimenten, luego se abre la válvula para que el agua pase al filtro y quede libre de residuos para ser reutilizada. Después todo el proceso anterior se hace el lavado del tanque 1 y los residuos son acumulados en el tanque 2 .

\section{Diagrama de flujo del sistema}
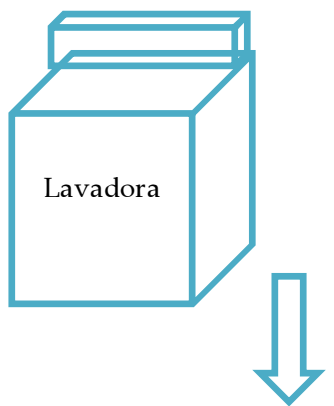

Agua

residual

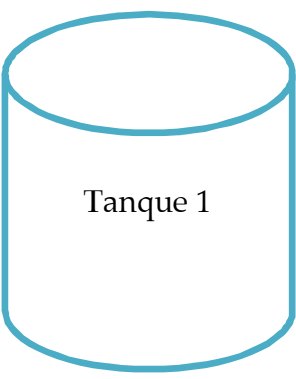

Residuos
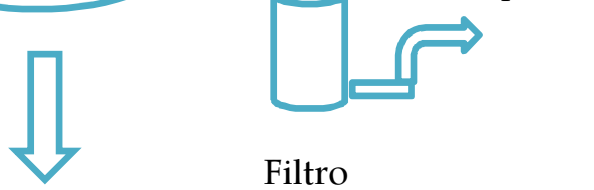

Filtro

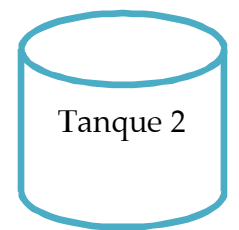

Figura 1: Diagrama de flujo del sistema.

\section{Diseño del sistema}

Para llevar a cabo el diseño del sistema se tuvo en cuenta una serie de parámetros que contribuyen a la recolección, tratamiento y reutilización del agua, los cuales son:

\subsection{Almacenamiento del agua residual}

Se contará con un tanque de almacenamiento que tenga capacidad de 20 litros, cuyo llenado se hará directamente desde el tubo de drenaje de la lavadora. El agua permanecerá en reposo durante 24 horas. 


\subsection{Coagulación}

Para la sedimentación se utilizará como coagulante el alumbre; con una dosificación de unos 20 a $50 \mathrm{~g}$ para el tratamiento.

\subsection{Mezclado}

Se utilizará como agitador un ángulo de aluminio para mantener la mezcla lo más homogénea posible y controlada, revolviendo de manera circular y vigorosamente durante tres a cinco minutos.

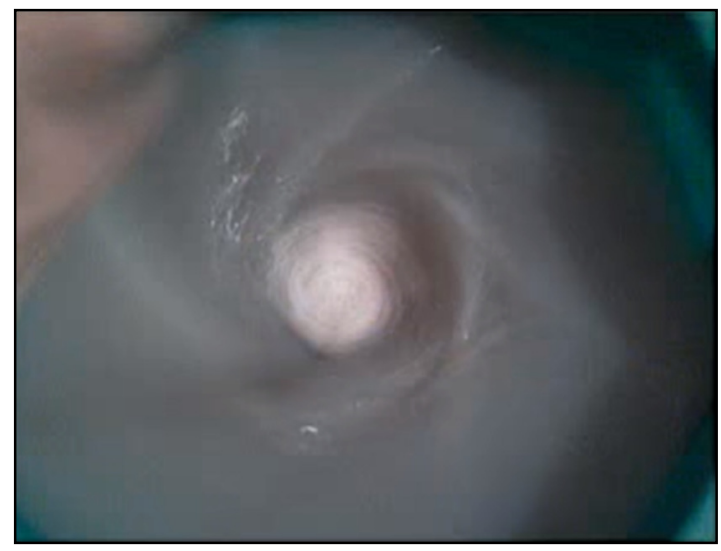

Figura 2. Mezclado del coagulante con el agua residual.

\subsection{Tiempo de tratamiento}

Se deja sedimentar, luego del mezclado del agua con el coagulante, aproximadamente 30 a 60 minutos.

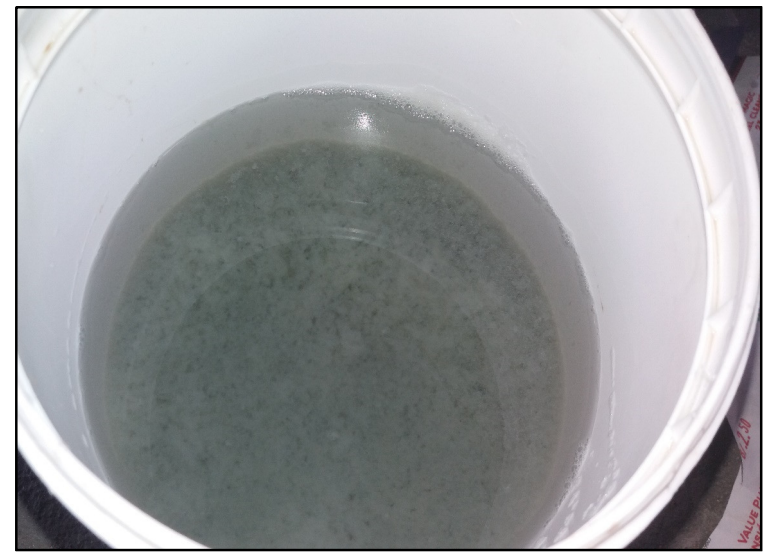

Figura 3. Sedimentación de los residuos.

\subsection{Filtración}

El tipo de filtro utilizado es de carbón activado, con una altura de $30 \mathrm{~cm}$ y diámetro de cuatro pulgadas. El filtro está compuesto por carbón vegetal, algodón y rejilla de plástico.

Para la limpieza del filtro se realizó un cambio, tanto del algodón como el carbón vegetal y otra opción sería el lavado del carbón y reemplazo del algodón [5].

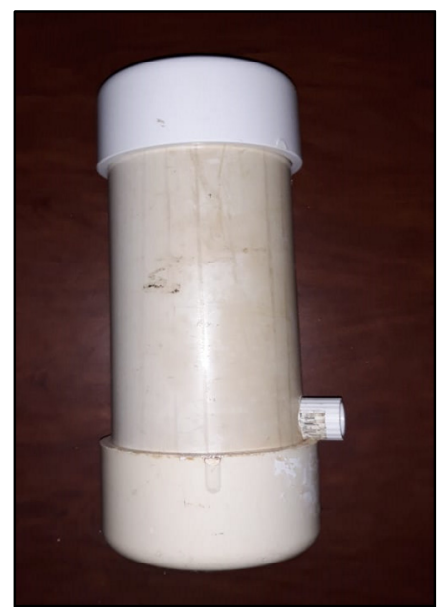

Figura 4. Filtro de carbón.

\subsection{Transporte del agua}

El transporte del agua desde el tanque de almacenamiento de agua residual hasta el filtro y del filtro a la llave, se dio a través de tubos PVC de 1/2".

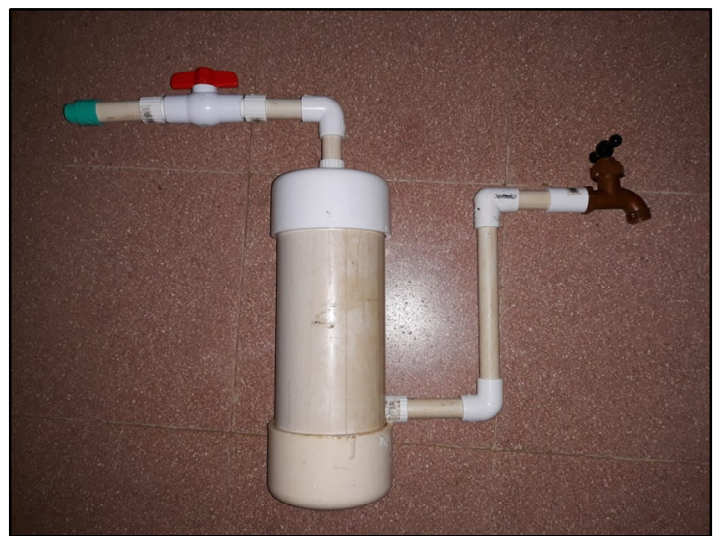

Figura 5. Tubería de transporte del agua.

\subsection{Lavado del tanque}

Se armó un tipo de drenado debajo del tanque de almacenamiento, mediante tubería con válvula, el cual permite evacuar los residuos y almacenarlos en otro compartimiento, quedando el sistema listo para su siguiente uso.

\section{Beneficios}

Mediante la ejecución del sistema de tratamiento de agua residual de lavadora se obtienen beneficios ambientales, ya que se espera disminuir los efectos que generan estas aguas al medio ambiente; y también se obtendrán beneficios económicos, es decir, tener un ahorro en el consumo del agua en los hogares, ya que el agua obtenida se reutiliza para un siguiente proceso de lavado, así como otros usos que no sean de consumo humano. 


\section{Resultados}

Como resultado después de los procesos por los que pasa el agua residual de coagulación, floculación y filtrado; se obtiene el agua limpia sin residuos que será reutilizada.

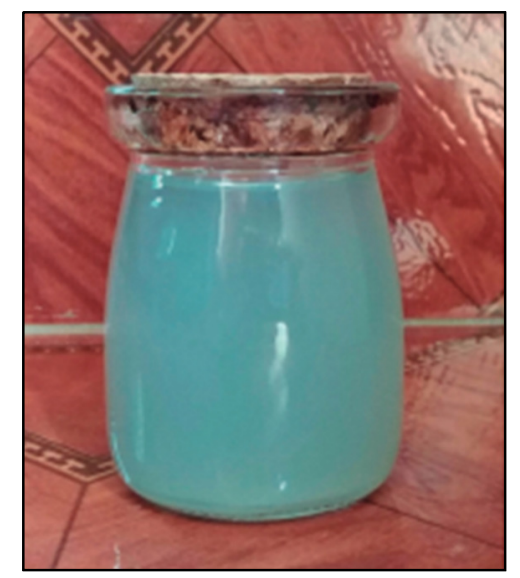

Figura 6. Agua residual almacenada.

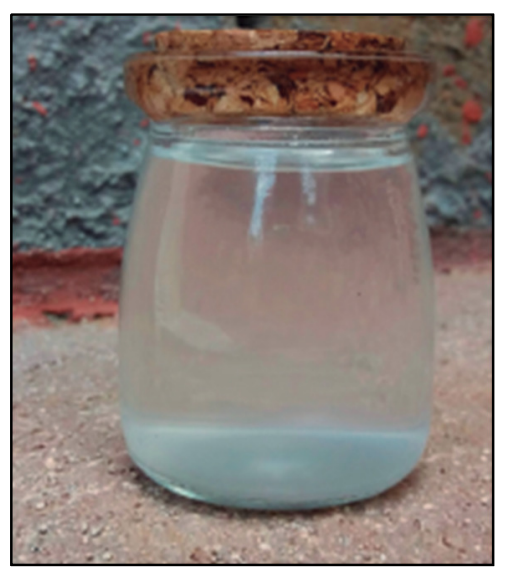

Figura 7. El agua luego del proceso de coagulación/floculación.

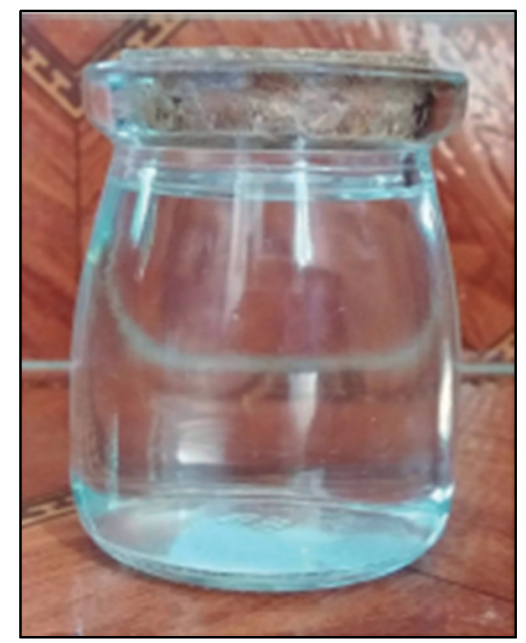

Figura 8. El agua luego de ser filtrada y lista para ser reutilizada

\section{Conclusiones}

- Mediante este sistema se puede reducir el consumo del agua en el hogar ya que se reutiliza a parte del proceso de lavado, en otras actividades.

- El sistema de tratamiento y reutilización de agua residual, es un sistema fácil de construir y a un costo moderado.

- La reutilización del agua es un mecanismo que beneficia el medio ambiente.

- Un limitante con el que cuenta el sistema diseñado es el tamaño, ya que depende de la disposición de espacio con el que se cuente en los hogares.

- El agua obtenida no es apta para el consumo humano.

\section{AGRADECIMIENTO}

Primero que todo agradecemos a Dios por brindarnos la sabiduría y salud en poder desarrollar este proyecto, a nuestras familias y a nuestro profesor asesor Fabio Velarde.

\section{REFERENCIAS}

[1] "Tratamiento de aguas residuales", Es.wikipedia.org, 2018. [Online]. Available: https://es.wikipedia.org/wiki/Tratamiento_de_aguas_residuales. [Ultimo acceso: 01- Jun- 2018].

[2] "U5. Sedimentación: General", Cidta.usal.es, 2018. [Online]. Available:

http://cidta.usal.es/cursos/agua/modulos/Conceptos/uni_04/u5c3 s1.htm\#Anchor2. [Ultimo acceso: 09- Jun- 2018].

[3] Y. Andía, «Tratamiento de agua - coagulación y floculación Sedapal,» Abril 2000. [En línea]. Available: http://www.sedapal.com.pe/c/document_library/get_file?uuid=2 792d3e3-59b7-4b9e-ae55-56209841d9b8\& groupId=10154. [Último acceso: 9 Junio 2018].

[4] Y. Andía, «Tratamiento de agua - coagulación y floculación Sedapal,» Abril 2000. [En línea]. Available: http://www.sedapal.com.pe/c/document_library/get_file?uuid=2 792d3e3-59b7-4b9e-ae55-56209841d9b8\&groupId=10154. [Último acceso: 9 Junio 2018].

[5] Carbotecnia, «Carbotecnia,» 7 Julio 2014. [En línea]. Available: https://www.carbotecnia.info/encyclopedia/que-es-el-carbonactivado/. [Último acceso: 9 Junio 2018]. 